\title{
Dépôt et calibration de multicouches pour l'optique XUV dans la gamme $10-30 \mathrm{~nm}$
}

\author{
F. Delmotte, M.F. Ravet, F. Bridou, X. Song, A. Jerome, S. Boujdayane, \\ S. Hubert ${ }^{1}$, Ph. Zeitoun ${ }^{1}$, M. Idir ${ }^{1}$ et P. Troussel ${ }^{2}$
}

Laboratoire Charles Fabry de l'Institut d'Optique, UMR 8501 du CNRS, bâtiment 503, Centre Scientifique d'Orsay, 91403 Orsay cedex, France

${ }^{1}$ Laboratoire d'Interaction du Rayonnement $X$ avec la Matière, LOA-ENSTA, Chemin de la Hunière, 91761 Palaiseau cedex, France

${ }^{2}$ CEA-DAM/DRIF/DCRE/SDE, BP. 12, 91680 Bruyères-le-Châtel, France

\begin{abstract}
Résumé.
Nous présentons dans une première partie une étude de miroirs interférentiels multicouches composés de divers matériaux pour des longueurs d'onde de l'ordre de $30 \mathrm{~nm}$. Les quatre systèmes étudiés, $\mathrm{Mo} / \mathrm{Si}$ recouvert de $\mathrm{Si}$ ou de $\mathrm{B}_{4} \mathrm{C}, \mathrm{B}_{4} \mathrm{C} / \mathrm{Si}$ et $\mathrm{Mo} / \mathrm{B}_{4} \mathrm{C} / \mathrm{Si} / \mathrm{B}_{4} \mathrm{C}$, présentent des pouvoirs réflecteurs de l'ordre de $24 \%$ autour de 30 nm. Les multicouches $\mathrm{B}_{4} \mathrm{C} / \mathrm{Si}$ et $\mathrm{Mo} / \mathrm{B}_{4} \mathrm{C} / \mathrm{Si} / \mathrm{B}_{4} \mathrm{C}$ présentent une meilleure sélectivité que les multicouches $\mathrm{Mo} / \mathrm{Si}$. Sur ces dernières, la couche de protection en $\mathrm{B}_{4} \mathrm{C}$ ne permet pas d'améliorer la stabilité en température, par contre l'ajout de $\mathrm{B}_{4} \mathrm{C}$ aux interfaces améliore considérablement cette stabilité.

Dans une seconde partie, nous présentons la réalisation et la calibration de lames séparatrices à 13,9 nm pour un interféromètre de Michelson. Les lames séparatrices que nous avons développées présentent des propriétés symétriques : les réflectivités de chaque face ne differrent que de $6 \%$. Le produit réflectivité - transmission de ces lames peut être optimisé au-dessus de $2 \%$ pour une longueur d'onde fixée dans la gamme $12,6-15,5 \mathrm{~nm}$.
\end{abstract}

\section{Introduction}

Les miroirs interférentiels multicouches, dont le principe est basé sur le dépôt alterné de couches minces (de 1 à $15 \mathrm{~nm}$ d'épaisseur), présentent aujourd'hui un intérêt tout particulier lié d'une part au développement de la lithographie EUV et des nouvelles sources de rayons X (rayonnement synchrotron, lasers à plasma, lasers à rayons $\mathrm{X}$, génération d'harmoniques) et d'autre part, au besoin en diagnostics de plasmas chauds (observation du soleil ou des plasmas créés par lasers de puissance). Fortement impliqué dans ces thèmes, le groupe de Physique des Films Minces du Laboratoire Charles Fabry de l'Institut d'Optique a notamment réalisé le télescope EIT (Extreme-ultraviolet Imaging Telescope) [1], embarqué dans le cadre de la mission SOHO (NASA/ESA) et est en charge des dépôts de multicouches pour la réalisation de l'imageur EUV de la mission STEREO (NASA).

Dans ce contexte, nos deux principaux axes de recherches sont d'une part l'étude de nouveau matériaux [2] et de nouvelles structures de multicouches multimatériaux (c'est à dire composées de plus de deux matériaux), et d'autre part le développement de nouveaux composants optiques, notamment d'optique XUV en transmission [3]. Après un bref descriptif des moyens expérimentaux utilisés (section 2), nous présenterons une étude de multicouches composées de divers matériaux pour des longueurs d'onde de l'ordre de $30 \mathrm{~nm}$ (section 3). Enfin, dans la section 4, la réalisation et la calibration de lames séparatrices à $13.9 \mathrm{~nm}$ pour un interféromètre de Michelson sera présentée.

\section{Dispositif expérimental}

Les multicouches sont déposées dans un bâti de pulvérisation par faisceaux d'ions qui permet de réaliser des structures comportant trois matériaux différents (figure 1). Ce bâti est constitué d'une enceinte ultravide $\left(\mathrm{P}<10^{-8} \mathrm{mbar}\right)$ équipée d'un canon à ions et d'un neutraliseur à cathode creuse. Les épaisseurs déposées sont contrôlées in-situ par réflectométrie X à 4,47 nm ainsi que par une microbalance à quartz. 
La pureté et la densité des matériaux déposés sont contrôlés ex-situ par réflectométrie des rayons $\mathrm{X}$ rasants à $0.154 \mathrm{~nm}$ en comparant les indices optiques calculés aux valeurs tabulées [4]. Les rugosités de surface et aux interfaces sont mesurées par réflectométrie et par microscopie à force atomique (AFM). Elles sont typiquement de l'ordre de $0,5 \mathrm{~nm}$.

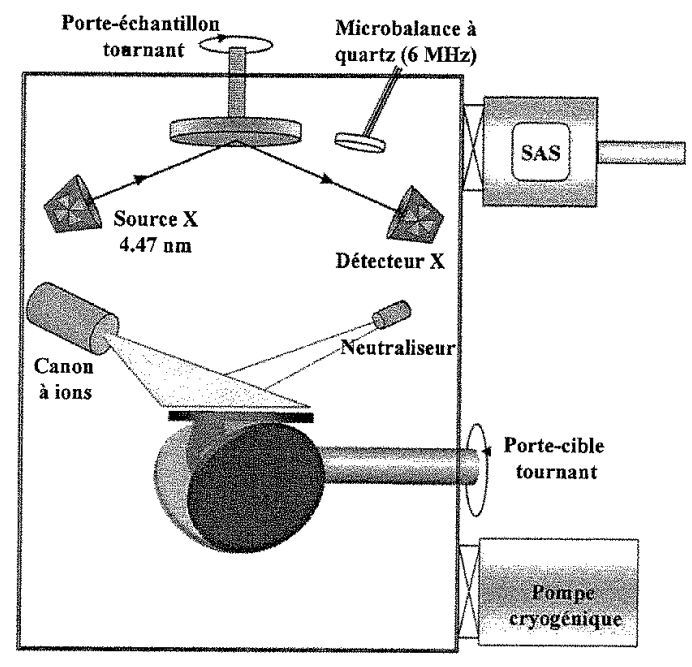

Figure 1, Schéma du bâti de dépôt de multicouches

3. Nouvelles multicouches pour des longueurs d'onde de l'ordre de $30 \mathrm{~nm}$

Nous avons mené une étude expérimentale de multicouches composées de divers matériaux pour des longueurs d'onde de l'ordre de $30 \mathrm{~nm}$ : Mo/Si recouverte de $\mathrm{Si}$ ou de $\mathrm{B}_{4} \mathrm{C}, \mathrm{B}_{4} \mathrm{C} / \mathrm{Si}$ et $\mathrm{Mo} / \mathrm{B}_{4} \mathrm{C} / \mathrm{Si} / \mathrm{B}_{4} \mathrm{C}$. Les deux principaux objectifs de ces nouvelles structures sont d'une part d'augmenter la stabilité des multicouches fabriquées et d'autre part d'améliorer leur sélectivité, tout en conservant, dans les deux cas, une réflectivité élevée.

Après avoir optimisé les paramètres de ces multicouches au moyen de simulations, les empilements ont été déposés et leur pouvoir réflecteur mesuré sur rayonnement synchrotron (ligne SA62, LURE-IAS,

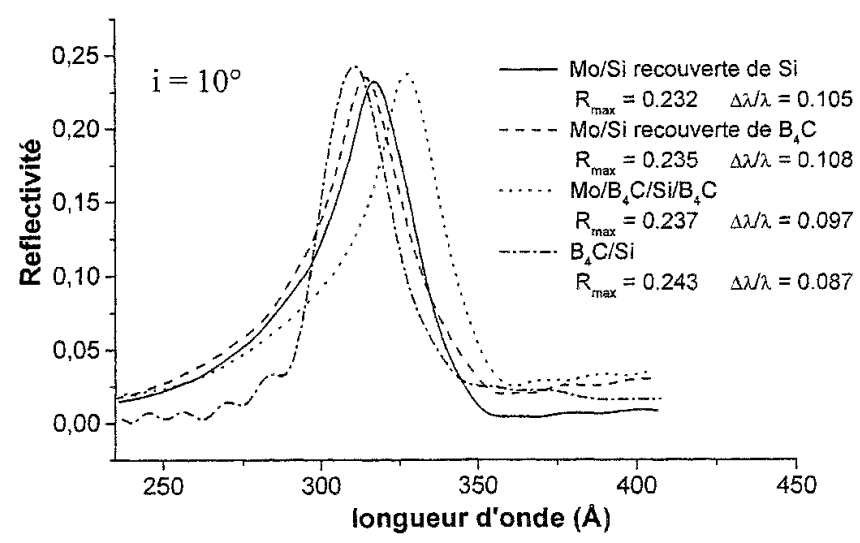

Figure 2. Mesures sur Rayonnement Synchrotron de différents empilements (multicouches de 15 périodes) 
Orsay) sous $10^{\circ}$ d'incidence. Les résultats obtenus sont tracés sur la figure 2 et montrent que les quatre systèmes étudiés présentent des pouvoirs réflecteurs de l'ordre de $24 \%$ autour de $30 \mathrm{~nm}$. Ces résultats expérimentaux montrent également que la sélectivité $(\Delta \lambda / \lambda)$ des multicouches $\mathrm{B}_{4} \mathrm{C} / \mathrm{Si}$ et $\mathrm{Mo} / \mathrm{B}_{4} \mathrm{C} / \mathrm{Si} / \mathrm{B}_{4} \mathrm{C}$ est meilleure que celle des multicouches $\mathrm{Mo} / \mathrm{Si}$. La valeur la plus faible de $\Delta \lambda / \lambda$ est obtenue pour une multicouche $\mathrm{B}_{4} \mathrm{C} / \mathrm{Si}$ et vaut $8,7 \%$ (pour $\lambda \approx 30,4 \mathrm{~nm}$ ).

Nous avons soumis ces quatre systèmes des recuits rapides ( 30 secondes sous air) à différentes températures afin d'étudier et de comparer leur stabilité en température. Sur la figure 3 , nous avons tracé la variation relative de la période $p$ de la multicouche ( $[p$ après recuit - $p$ avant recuit $]$ / $p$ avant recuit) en fonction de la température. La couche de protection en $\mathrm{B}_{4} \mathrm{C}$ ne permet pas d'améliorer la stabilité en température des multicouches $\mathrm{Mo} / \mathrm{Si}$, par contre l'ajout de $\mathrm{B}_{4} \mathrm{C}$ aux interfaces améliore considérablement cette stabilité.

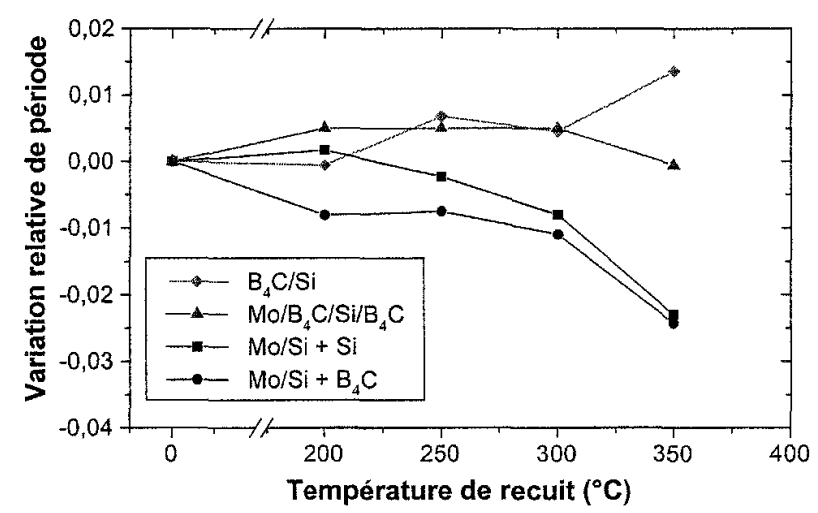

Figure 3. Variation relative de période des multicouches après un recuit rapide de $30 \mathrm{~s}$.

\section{Lames séparatrices pour l'interférométrie sur laser X}

Le but de cette étude était de concevoir des lames séparatrices pour un interféromètre de Michelson sur laser $\mathrm{X}$ à $89,2 \mathrm{eV}(13,9 \mathrm{~nm})$. La fabrication de telles optiques nécessite le dépôt d'une multicouche parfaitement calibrée sur un support extrêmement mince (une membrane d'épaisseur inférieure à $100 \mathrm{~nm}$ ). Compte tenu des fortes contraintes mécaniques en compression induite par le dépôt en pulvérisation par faisceau d'ions, nous avons choisi de déposer des multicouches sur des membranes de nitrure de silicium fortement tendues de manière à conserver une planéité acceptable après le dépôt. Dans un interféromètre de Michelson, les faisceaux lumineux suivant les deux bras ne se réfléchissent pas sur la même face de la lame séparatrice. Nous avons donc conçu des lames séparatrices symétriques en déposant des multicouches Mo/Si sur les deux faces d'une membrane de $\operatorname{SiN}$ (ouverture $=5 \times 5 \mathrm{~mm}$, épaisseur $\approx 90 \mathrm{~nm}$ ).

Les paramètres des multicouches $\mathrm{Mo} / \mathrm{Si}$ ont été calculés par simulation de manière à optimiser le produit ( $\mathrm{RxT}$ ) de la lame séparatrice. Ces multicouches ( 3 périodes de $10 \mathrm{~nm}$ ) ont été déposées sur chaque face de plusieurs membranes d'épaisseur comprises entre 80 et $100 \mathrm{~nm}$. Les réflectivités et transmission de chaque face ont ensuite été mesurées à $45^{\circ}$ d'incidence sur rayonnement synchrotron (ligne SA23, LURE, Orsay).

Les lames séparatrices que nous avons développées présentent des propriétés symétriques : les réflectivités de chaque face ne diffërent que de 6\% (figure 4). La différence de rugosité entre les faces avant et arrière de la membrane SiN (respectivement $0,5 \mathrm{~nm}$ et $0,1 \mathrm{~nm}$ en rugosité rms mesurée par AFM) permet d'expliquer cette différence de réflectivité. Comme le montre la figure 5 , pour une énergie de photons fixée dans la gamme $80-98 \mathrm{eV}(12,6-15,5 \mathrm{~nm})$, il est possible d'optimiser le produit RxT de ces 
lames séparatrices en faisant varier l'épaisseur de la membrane SiN. On obtient des valeurs supérieures à $2 \%$ pour une polarisation s sur cette gamme d'énergie.

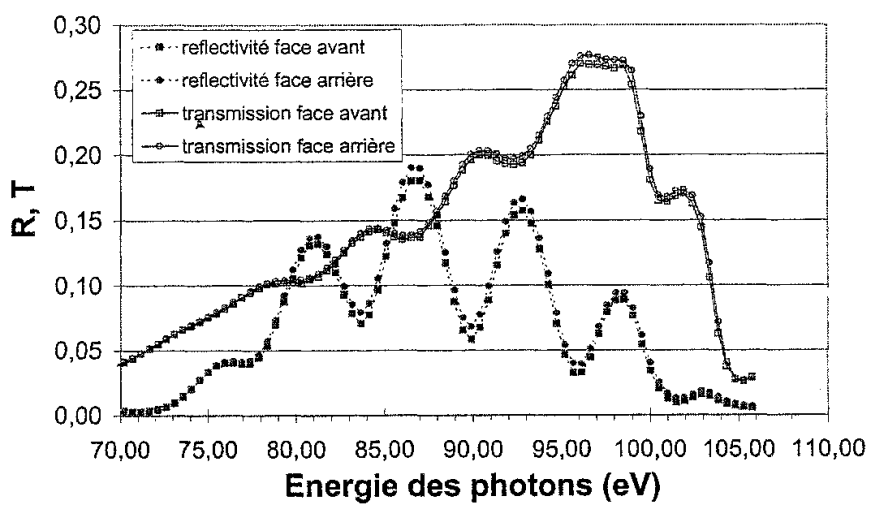

Figure 4. Mesures sur Rayonnement Synchrotron de la réflectivité $R$ et de la transmission $T$ d'une lame séparatrice sur chaque face. Lame $\mathrm{n}^{\circ} 7 \mathrm{a}$ (épaisseur $\mathrm{SiN}=84.9 \mathrm{~nm}$ ).

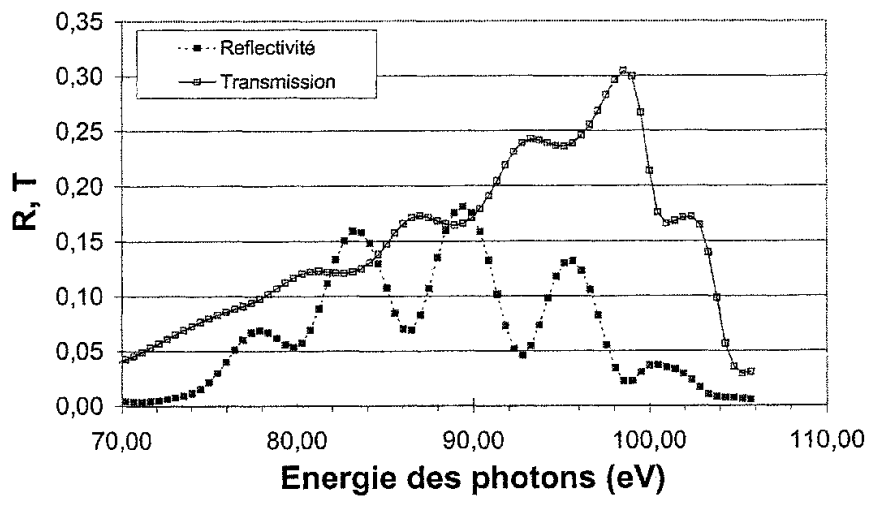

Figure 5. Mesures sur Rayonnement Synchrotron de la réflectivité $R$ et de la transmission $T$ d'une lame séparatrice optimisée pour $89.2 \mathrm{eV}$. Lame $\mathrm{n}^{\circ} 7 \mathrm{c}$ (épaisseur $\mathrm{SiN}=80.3 \mathrm{~nm}$ ).

\section{Références}

[1] Delaboudinière J.P., Artzner G.E., Brunaud J., Gabriel A.H., Hochedez J.F., Miller F., Song X.Y., Au B., Dere K.P., Howard R.A., Kreplin R., Michels D.J., Moses J.D., Defise J.M., Jamar C., Rochus P., Chauvineau J.P., Marioge J.P., Catura R.C., Lemen J.R., Shing L., Stern R.A., Gurman J.B., Neupert W.M., Maucherat A., Clette F., Cugnon P., Van Dessel E.L., Solar Physics 162 (1995), 291-312.

[2] Ravet M.F., Bridou F., Raynal A., Pardo B., Chauvineau J.-P., André J.-M., J. Appl. Phys. 89 (2001), $1145-1150$.

[3] Delmotte F., Ravet M.F., Bridou F., Varniere F., Zeitoun P., Hubert S., Vanbostal L., Soullie G., XUV beamsplitters for Michelson interferometer, to be published in Applied Optics.

[4] Bridou F., Pardo B., J. Optics (Paris) 21 (1990), 183-191. 